\title{
Fuzzy Based Analysis for Efficient Clustering in MANET and Security Enhancement Approach using Neural Network
}

\author{
V. Preetha ${ }^{1}$, K. Chitra ${ }^{2}$ \\ ${ }^{1}$ Department of Computer Science, Bharathiar University \\ ${ }^{2}$ Department of Computer Science, Govt Arts College, Melur Madurai \\ India
}

\begin{abstract}
Mobile Adhoc Network has become an essential one in every aspects of our life due to the recent growth of technological developments. Effective communication in right scenarios is an important factor to be considered. This paper is divided into two parts. In the first part Cluster based routing for a specific disaster scenario and the parametric analysis for the effective clustering in MANET is analyzed and proposed using fuzzy logic with mat lab simulator and the second part focuses on the security Enhancement using Neural Network approach.
\end{abstract}

\section{Introduction}

A mobile ad hoc network consists of a group of wireless-enabled devices. Initially, it was named as packet radio network and was initiated by the Department of Defense (DoD) of the United States of America. Mobile adhoc network itself is an autonomous system consisting of collection of mobile hosts connected by wireless links. It has no supporting fixed infrastructure or central administration and the nodes will communicate within the transmission range of each other. If two hosts are unable to communicate, then they will try to communicate if other hosts lying in between are willing to forward packets for them. Thus every node will participate in multi-hop routing to reach all the nodes in the network. Continuous improvement in smart devices in wireless world has gained the interest of the users in capitalizing these smart devices. MANET is widely used in rescue operations, military scenarios and in scenarios where it is impossible to establish a wired backbone. While considering the emergency situation particularly in mass disasters, a quick and coordinated response must be given to improve the efficiency of rescue teams and to save as many lives as possible. Furthermore, the emergency situation may be ongoing for some time or even days; hence systems may have to stay usable for extended periods. This is the case of IMPROVISA (Improvisa) that proposes to solve this difficulty by distributing antennas in the disaster area [1]. Some other applications of
MANET include the adhoc network created in smart classrooms by the students and Professors with the laptops and by a group of people at a meeting using their PDAs or laptops to exchange information among themselves. In future, MANET will be widely used in ubiquitous computing, providing connectivity to everyone, anywhere and from any device. As a result, robust routing mechanisms and improving the Quality of service becomes inevitable. Mobility of nodes in MANET and limited battery power, dynamic topology changes, link bandwidth are major challenges in MANET when concerned with routing, scalability and management functions.

\section{Hierarchical Routing in Emergency Scenarios}

Mobile ahoc network undergoes multihop network topology that may change frequently due to mobility, congestion in traffic, power constrained problems and computational overhead. Many routing protocols are set up for efficient routing. Proactive routing, reactive routing, Hybrid routing protocols and the route constructed are kept alive as long as possible. Location aided routing protocol is based on the nodes location information. The communication overhead of proactive protocols is $o\left(n^{2}\right)$ where n represents the total number of mobile terminals [2]. Reactive routing protocols faces scalability and mobility challenges. Hybrid protocols exhibit both reactive and proactive features. The drawbacks of proactive and reactive protocols such as overheads and delay are minimized in Hybrid routing. A hierarchical routing depends on the hierarchic level in which a node resides. For the better performance of MANETs a hierarchical architecture is essential.

\section{Emergency Rescue Scenarios}

MANET is widely used in Emergency rescue scenarios, Disaster recovery, supporting doctors and nurses in hospitals, Environmental disasters etc. Hence in such cases continuous evaluation of the routes between the routes is very important to rescue the people affected. In case of emergency and rescue scenario, there may be obstacles which affect the normal mobility pathways. Many network providers 
are providing instant networks in disaster areas with mobile base stations, antennas and power generators. These instant networks will be lifesaving because the deployment of placing a fixed network will take much duration and the cost will be high. When Typhoon Bopha hit the Philippines an instant network was created and many people were rescued. While analyzing the emergency scenarios, zone based link connectivity and mobility are the important factors to be considered. In order to overcome the disadvantages of reactive and proactive hierarchical structure has been developed. In general, Cluster based routing protocol [CBRP] falls under the reactive category but due to its leveloriented administration and governance by cluster head it has the hierarchical component. CBRP has many advantages such as energy consumption and network performance. Thus a typical hierarchical structure can be implemented by partitioning the network into clusters depending on the geographic region, transmission range, and communication reliability irrespective of the sparse and dense regions.

\section{Clustering Scheme}

Clustering refers to the partitioning of the network in to different sizes. Transmission range and Zone based partitioning will aid in the easier routing. Liliana Enciso Quispe et.al analyzed the hierarchical routing in their work based on the Behavior of Ad Hoc routing protocols, analyzed for emergency and rescue scenarios, on a real urban area[3] and suggests that the use of CBRP protocol in a disaster area, more efficiently adjusts the evacuation of persons and their care and appropriate location. CBRP routing will be very efficient in the case of rescue operations for optimum routes, large scalability, traffic reduction etc.

\section{Disaster Area Model and Problem Specification}

This paper focuses on the following disaster area model with network connectivity. This temporarily created MANET involves mainly communication devices for effective communication. This may include even vehicular adhoc nework, Air network, fly network with mobile base stations. Clustering in this type of MANET will be very suitable for effective communication. This may save the energy of the resources and for fastest telecasting and monitoring of the current situation. The Government Hospitals, Police station and Telecasting media centre may be at a particular distance. Connectivity index can be used to find the shortest path.

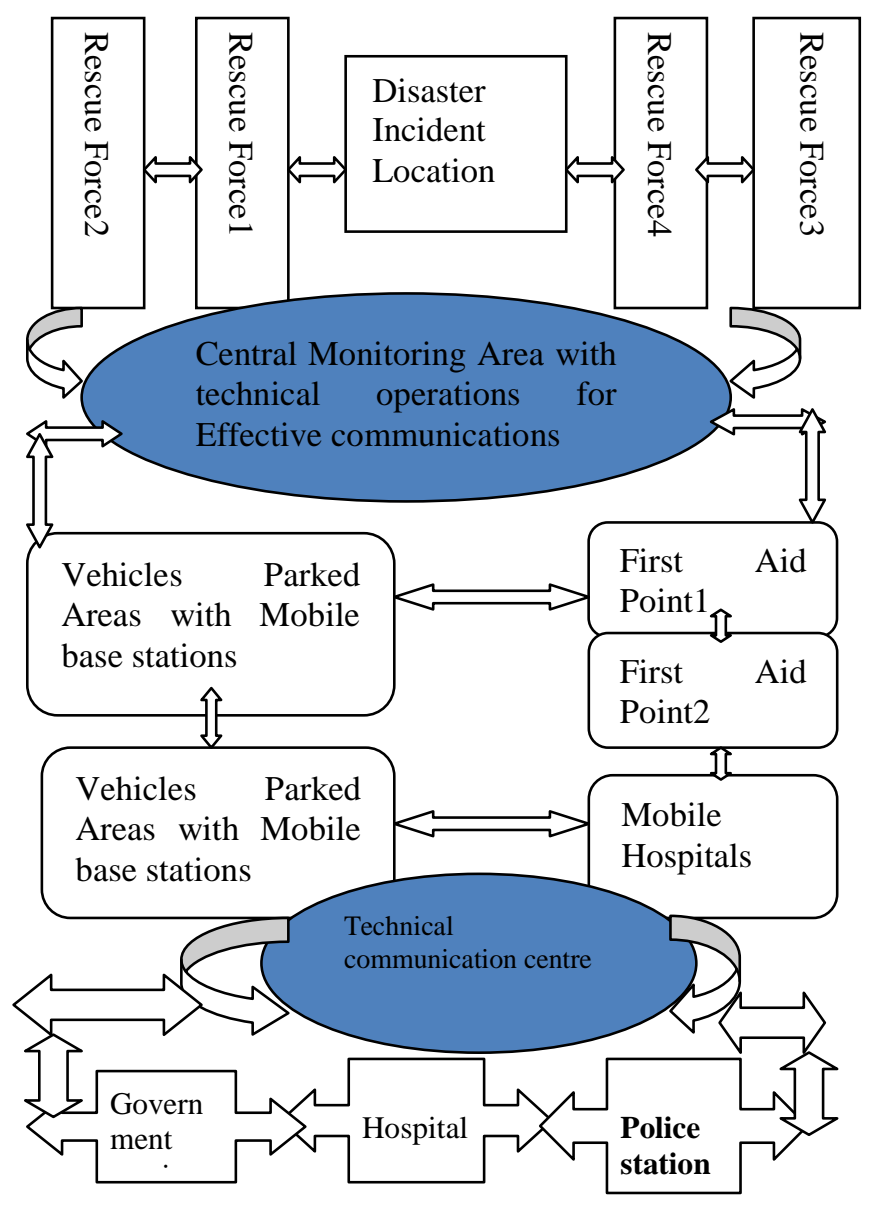

Figure 1. Disaster Area Model

We are analyzing some of the following necessary parameters for the effective chance of clustering in MANET.

\section{Parameter analysis using fuzzy logic}

Different Parameters are essential for clustering in Manet.The following very important parameters are taken into consideration for effective clustering:

\subsection{Connectivity Index}

The first Parameter chosen is connectivity Index. Connectivity Index is an important parameter while considering the Emergency Rescue scenarios. The following picture illustrates the connectivity Index. 


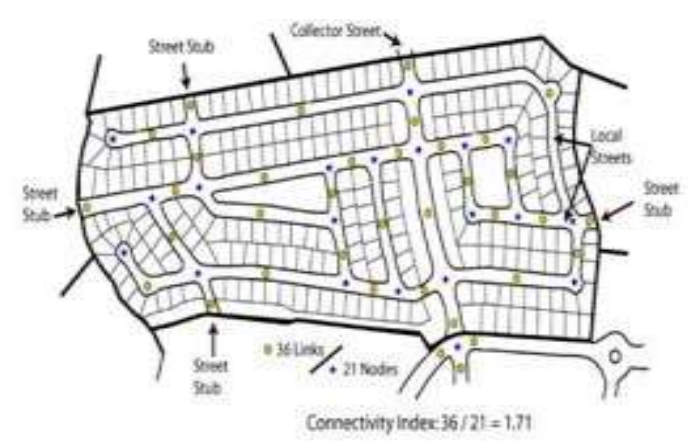

Figure 2. Connectivity Index of the City of Fayetteville, North Carolina

The above Connectivity index represents the area in the the City of Fayetteville, North Carolina [3]. Connectivity Index is also Known as Randić index. It was proposed by Randić in 1975. [4] $\chi(\mathrm{G})$ is defined as the connectivity index and it was calculated based on the degree of vertex. In general, Connectivity index is also an important entity when analyzing the MANET implementation in the above disaster scenarios. Nodes (stars) exist at street intersections.Links (circles) are represented as stretches of road that connect nodes. In the above diagram, there are 36 links (circles) and 21 nodes (stars); therefore the connectivity index is 1.71 $(36 / 21=1.71)$. The greater connectivity index factor shows the links between the source and destination for easier reachability and routing. By assuming that if the disaster area is divided into zones as central zone,zone1,zone2,zone3 etc.If zone1 and Zone2 recovery process was finished, the links can be added or deleted and connectivity can be redirected. In some Connectivity analysis problems, the connectivity is defined by the probability that a node is reachable at any other node in the network. For a single component graph, any node is reachable at other nodes, thus, the connectivity is equal to 1 .So connectivity can be defined as

$$
\text { connectivity }=\frac{\mid \text { largest connected component } \mid}{\mathrm{N}}
$$

Where $\mathrm{N}$ is the total number of nodes in the network.

\subsection{Mobility}

For this scenario, the assumption is that the mobility of the nodes will not be too fast .It depends on the incident area zone. Many mobility models have been described by authors. Some of them are real life mobility models, models based on topology restrictions and statistical models with random mobility. Different mobility metrics with relative speed velocity are calculated based on the position of the nodes. But in a Disaster area scenario GPS system will be helpful for identifying the position of the nodes even though the hardware cost is high and the energy consumption is high.

\subsection{Transmission Range}

The transmission range may vary from short range to long range. The disaster scenarios will have the transmission range depending on the incident location and the density of the nodes. A larger transmission range will have greater connectivity. The total number of nodes within the transmission range is defined as:

$$
\sum_{\mathrm{u} \in \mathrm{v}, \mathrm{u} \neq \mathrm{v}}\left\{\mathrm{D}_{\mathrm{uv}}<\mathrm{T}_{\mathrm{x},} \text {, Transmission range }\right\}
$$

\subsection{Density of the nodes}

If the density of the nodes is high there may be higher attenuation. Generally, the density of the nodes in the disaster area may depend on the population of the area.

\subsection{Battery energy}

Each mobile node has a defined amount of energy. The energy of the node is the important factor in communication devices. If this amount is low, the node can neither send nor receive any data. The energy consumption of a node during a network will have the following states:
a. Sleep mode
b. Idle mode
c. Transmit
d. Receive

Here in this disaster scenario, we are not considering the energy of a particular node to calculate the membership function. But the leader node has to be chosen for clustering based on highest energy.

\subsection{Fuzzy Logic}

The fuzzy logic was used to represent uncertainties. Fuzzy logic control system is rulebased system which contains a set of fuzzy rules. It will be very useful for control decision mechanism. Mamdani fuzzy-rule based systems have the linguistic description which has both the antecedent parts and the consequent parts. Rule base is an IFTHEN rule group with fuzzy sets that represents the desired behavior of a fuzzy system.

IF $\mathrm{x}_{1}$ is $\mathrm{A}_{\mathrm{i} 1}$ and ... and $\mathrm{x}_{\mathrm{n}}$ is $\mathrm{A}_{\mathrm{in}}$ THEN $\mathrm{y}$ is $\mathrm{C}_{\mathrm{i}}, \mathrm{i}$ $=1,2, . . \mathrm{L}$ 
Where $\mathrm{L}$ represents the number of fuzzy rules, $\mathrm{X}$ is the input variable, $y$ is the output variable. $A_{i j}$ are the fuzzy sets of the input linguistic variable $x_{j}$ and $c_{i}$ represents the set of the output linguistic variable $y$. $\mathrm{A}_{\mathrm{ij}}$ and $\mathrm{c}_{\mathrm{i}}$ are characterized by both membership functions. For the above discussed parameters, the fuzzy membership function is calculated and the effect of clustering in the above discussed disaster scenario MANET is discussed by rule-based system.

Table 1. Parameters chosen for clustering

\begin{tabular}{|c|c|}
\hline $\begin{array}{l}\text { Parameters } \\
\text { Chosen for } \\
\text { Clustering }\end{array}$ & Range \\
\hline \multirow{3}{*}{$\begin{array}{l}\text { Connectivity Index } \\
\text { of a particular } \\
\text { Zone }\end{array}$} & High \\
\hline & Medium \\
\hline & Low \\
\hline \multirow{3}{*}{$\begin{array}{l}\text { Transmission } \\
\text { Range }\end{array}$} & Long \\
\hline & Short \\
\hline & Medium \\
\hline \multirow{3}{*}{ Mobility } & High \\
\hline & Medium \\
\hline & Less \\
\hline \multirow{3}{*}{ Density } & Large \\
\hline & Medium \\
\hline & Small \\
\hline
\end{tabular}

The membership functions of the above parameters are derived using fuzzy logic in Mat lab as:

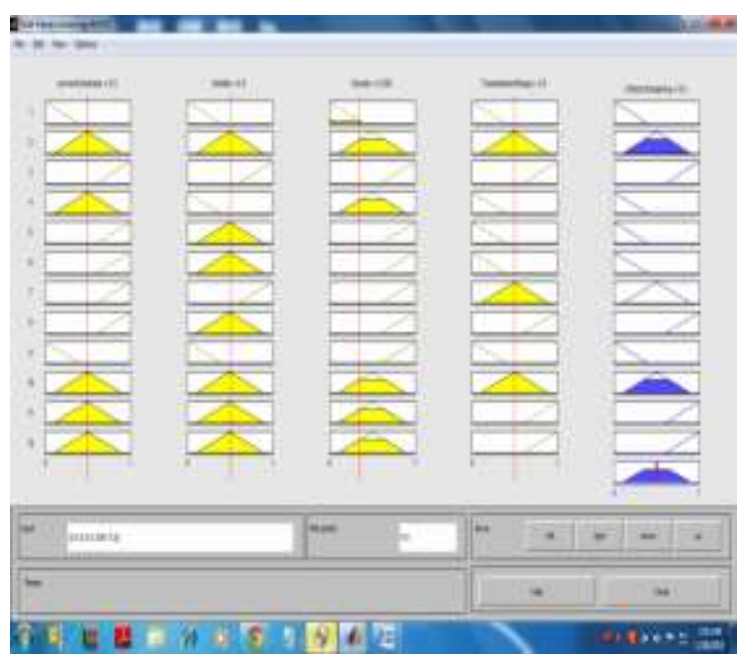

Figure 3. Membership functions for the selected parameters

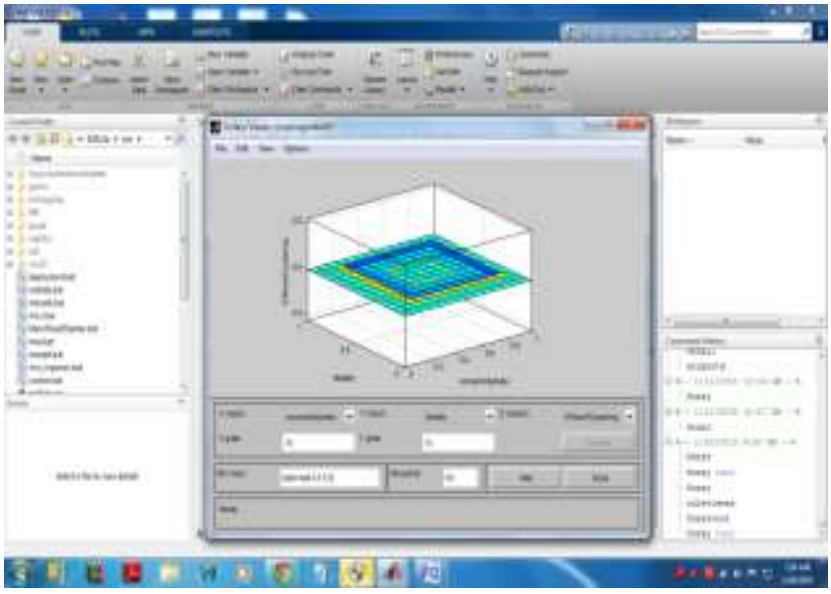

Figure 4. Parameter Analysis Graph for effective clustering

Thus the required important parameters for the clustering in MANET in case of disaster scenarios using fuzzy logic. The rules are generated for the chance of effective clustering in MANET using Rule-based Mamdani. This shows that if the parameters connectivity index hand the transmission range is high and if the mobility and density are medium then the chance of the effective clustering is best. The graph shows the impact of the parameters on the effective clustering for MANET.

\section{Security Enhancement using Neural Network Approach}

Neural Network is a learning technique to derive meaning from complicated or imprecise data, to extract patterns and to detect trends that are too complex to be noticed.The advantages of Neural network includes

$>$ Adaptive learning
$>$ Self-organization
$>$ Real-time operation
$>$ Fault tolerance via redundant
information coding

The training in Neural networks include Supervised, unsupervised and Reinforcement Training. A neural Network consists of the input layers, Hidden layers and output layers. Weight is information used to solve a problem. Since the Neural Network output will determine the probability the prediction can be done especially in case of attacks or malicious detection. In communication networks the output can be used for further testing by effective learning algorithms. The following figure represents the architecture of the Neural Network. 


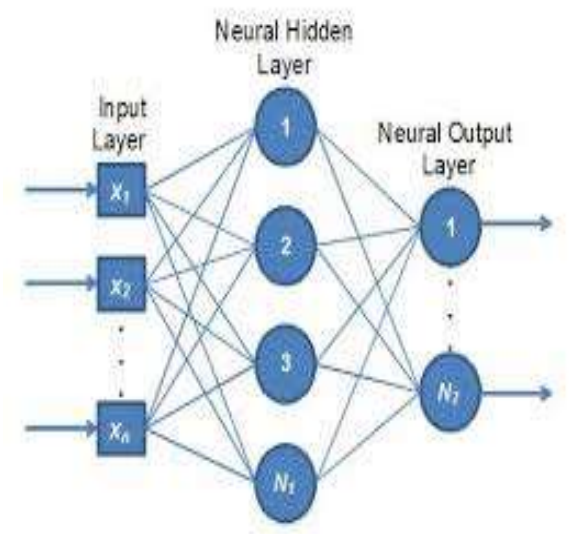

Figure 5. Neural Network Architecture

Once the Efficient clustering is formed the node authentication can be verified using Neural Network approach.The Initialized Neural Network must be trained with the appropriate training algorithm .The number of weights and biases in the network and the mean square error between the actual and desired network responses are taken into consideration.

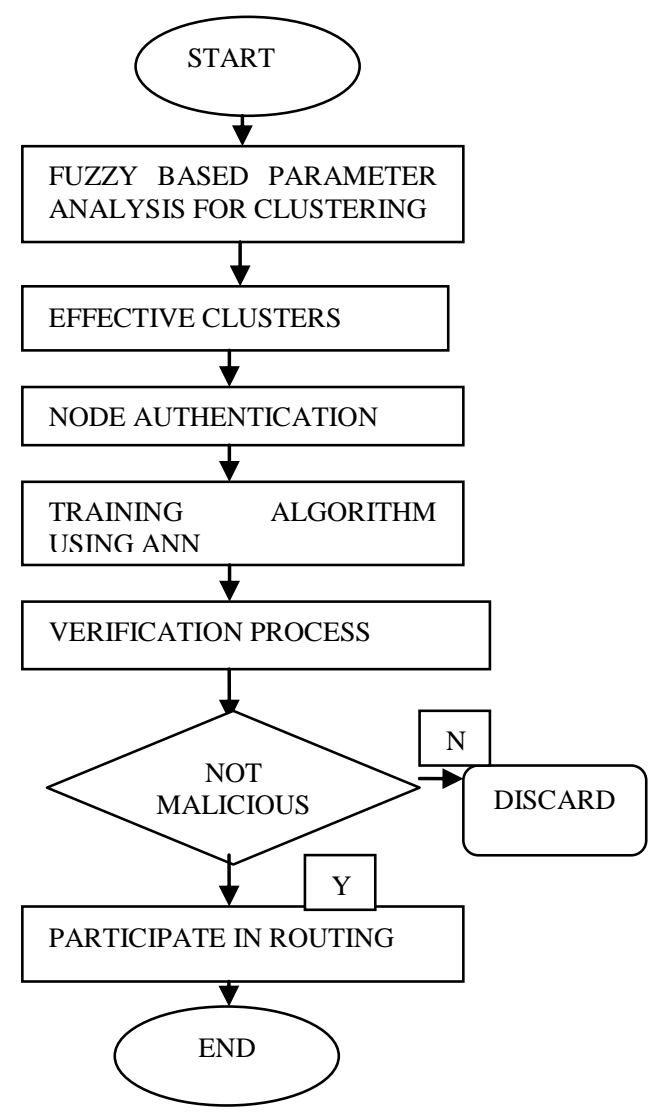

Figure 6. Proposed NN Approach

\section{Conclusion}

This paper focuses on the required important parameters for the effective clustering chance especially in focus of the disaster scenarios. It proves that the chance of effective clustering is possible in disaster scenarios and the important parameters are analyzed by focusing on efficient clustering. The security can be enhanced using the proposed Neural Network approach.

\section{References}

[1] Abraham Martın-Campillo, Jon Crowcroft, Eiko Yoneki, Ramon Mart1, "Evaluating opportunistic networks in disaster scenarios," Journal of Network and Computer Applications, 2013, pp. 870-880.

[2] Jane Y.Yu and Peter H.J. Chong, "A Survey of Clustering Schemes for Mobile adhoc networks", IEEE Communications Surveys \& Tutorials, vol. 7, 2005, pp. 32-47.

[3] M. A. Rajan, M. Girish Chandra,Lokanatha C. Reddy and Prakash Hiremath," A Study of Connectivity Index of Graph Relevant to Ad Hoc Networks", IJCSNS, International Journal of Innovative Research in Computer and communication Engineering. vol. 2, 2014, pp. 51525157.

[4] Liliana Enciso Quispe, Luis Mengual Galan," Behavior of Ad Hoc Routing protocols analysed for emergency and rescue scenarios, on a real urban area ", Expert systems with applications, 41.5 (2014): 2565-2573.

[5] Sharmila John Francis and Elijah Blessing Rajsingh,"Performance Analysis of Clustering protocols in Mobile Adhoc Networks", in Journal of Computer Science 4 (3), 2008, pp.192-204

[6] Mainak Chatterjee, Sajal K.Das and Damla Turgut, "WCA:Weighted Clustering Algorithm for Mobile Adhoc networks", in Cluster Computing 5,2002, pp.193-204.

[7] Charalampos Konstantopoulos, Damianos Gavalas, Grammati Pantziou, "clustering in Mobile Adhoc network through neighbourhood stability based mobility prediction", in Computer Networks, 2008,pp.1797-1824.

[8] Halenar Igor, Juhasova Bohuslava, Juhas Martin, Nesticky Martin, “Applications of Neural Networks in Computer Security" in Procedia Engineering, Elsevier, 2013, pp. 1209-1215. 